\title{
Take an Energy-saving And Comfortable Hot Bath
}

\author{
Yibin Zhou \\ School of North China Electric Power University, Baoding 071000, China; \\ 2212327403@qq.com
}

Keywords: Energy-saving, Comfortable,Hot Bath

\begin{abstract}
Bathtub is a kind of pipeline device used in bathroom or shower,which usually used in the family bathroom.Most modern bathtubs are make from strength (acrylic) or glass fiber,In the fast pace of life space, bathtub is a good way for people to cleanse and relax. In this paper, we only talk about the bathtub, which is a simple water containment vessel. And the excess water escapes through an overflow drain,what we need to do is how to add heating water to Bathtub to make people have been in a comfortable bath and We don't waste too much energy at the same time.
\end{abstract}

\section{Introduction}

A person lying in the bathtub adds hot water to keep the water warm. A lot of motions will be made by the person in the bathtub. In our problems, the motion changes the distribution of temperature field and causes the temperature field in the same temperature. We establish the temperature field of the time and space to describe the change of bathtub. We define "the constant trickle of hot water from the faucet to reheat the bathing water" as that the water drops into the bathtub at a uniform speed.

Our model describes the influence of hot water on the temperature field. Within the context of water conservation, we determine the optimum feed water temperature and the flow rate.

\section{Our model}

We put the bathtub into the Descartes coordinate system, establishing a Temperature Field Model of bathtub. For the convenience of calculation,we assume that the bathtub is a rectangle. We also simplify the human body to polyhedron.

We use ${ }^{X_{0}},{ }^{y_{0}}$ and $Z_{0}$ to express the length, width, and height of bathtub. At first, we do not fill the bathtub with water considering water conservation. In this way, hot water and cold water can be mixed more evenly in the process of following heating water. We assume that $t_{0}$ is initial temperature of water, ${ }^{t_{1}}$ is the water temperature when the human body obviously feels cold, and $t_{2}$ is the temperature of added water.We believe the constant trickle of hot water from the faucet is constant in per unit time. Based on the above content, it is not difficult to find that the whole system meets energy conservation law, conservation of momentum law, and continuity principle of fluids flow.

$\phi, \phi^{\prime}$ are heat flowing into and out of the surface in three directions. $\rho$ is density of water, ${ }^{C}$ is constant-pressure specific heat of water, ${ }^{t}$ is temperature, $a$ is thermal diffusivity of fluid, $\dot{\phi}$ is strength of internal heat source. We believe that the hot water into the bathtub will be mixed with cold water because of human motion, which equivalent that the water can produce heat. In other words, there is an inner heat source in the water.Assuming that $W$ is the current velocity added hot water, $A$ is the cross-sectional area of outfall, $V_{0}$ is the initial volume of water in the bathtub

$$
\begin{gathered}
d Q_{i n}=d m c\left(t_{1}+273\right) \\
d m=\rho W A d \tau
\end{gathered}
$$

The strength of internal heat source is defined as the heat generated in unit volume unit time ${ }^{[1]}$, so the strength of internal heat source

$$
\dot{\phi}=\frac{\rho W A c\left(t_{1}+273\right)}{V_{0}+\int \rho W A d \tau}
$$


We can list the continuity equation because the flow of water is continuous

$$
\frac{\partial t}{\partial x}+\frac{\partial t}{\partial y}+\frac{\partial t}{\partial z}=0
$$

We can get the energy differential equation through equation (1) and the mechanism of heat conduction and heat transfer

$$
\frac{\partial t}{\partial \tau}+u \frac{\partial t}{\partial x}+v \frac{\partial t}{\partial y}+w \frac{\partial t}{\partial z}=a\left(\frac{\partial^{2} t}{\partial x^{2}}+\frac{\partial^{2} t}{\partial y^{2}}+\frac{\partial^{2} t}{\partial z^{2}}\right)+\frac{\dot{\phi}}{\rho c}
$$

If we want to solve the temperature field,we need to know the velocity field of the fluid.

At the same time, we know a series of boundary conditions

$$
\begin{aligned}
& \left.\frac{\partial t}{\partial X}\right|_{X= \pm \frac{X 0}{2}}=0 \\
& \left.\frac{\partial t}{\partial Y}\right|_{Y=0, Y_{0}}=0 \\
& \left.\frac{\partial t}{\partial Z}\right|_{Z=0}=0
\end{aligned}
$$

The heat radiation of surface mainly consists of natural convection heat transfer,evaporative cooling, and thermal radiation ${ }^{[2]}$. For the convenience of calculation, we define a coefficient of heat transfer $\mathrm{K}$ again, that is called convective coefficient of heat transfer. We unify the three ways of heat transfer. We can use the experimental method or directly apply the empirical formula to obtain $\mathrm{K}$. This article will not be discussed in detail in this paper.

The boundary condition of the water surface is

$t_{a}$ is room temperature.

$$
-\left.\lambda \frac{\partial t}{\partial Z}\right|_{z}=Z_{0}=K\left(t-t_{a}\right)
$$

We regard the surface of the human body as a constant temperature; the added hot water is also a constant temperature. Then we have the boundary temperature value as a boundary condition

$t=t_{1} \mid X=0, Y=0, Z=Z_{0}$

On these surfaces, $t=t_{p}, t_{p}$ is the temperature human surface area.

The velocity gradients are 0 in the normal directions of walls because of liniment of bathtub walls. The mathematical description are

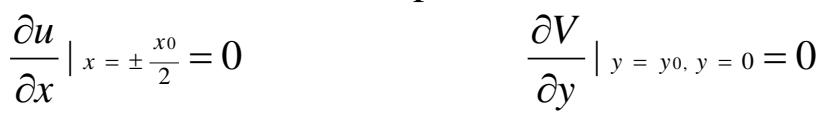

The speed of the contact point is equal to the speed of the faucet when the tap was hit by water (ignoring the impact of gravity on the flow rate)

$W=-W \mid x=0, y=0, z=z 0$

The water will rise in the process of adding water to bathtub

$$
\frac{\partial w}{\partial z}=\frac{W A}{x_{0} y_{0}}
$$

We can use mathematical method to describe an astable temperature field of a time and space by using the above equations and boundary conditions. The expression must be a function with regard to hot water temperature and the velocity of the flow $t=f\left(x, y, z, W, t_{1} \ldots\right)$

The model is establishing a model of an astable temperature field in the process of water injection. But the water will fill the bathtub when the time reaches certain moment. Assuming that the body is immobile, the bathtub becomes a steady system. Therefore, the temperature field of the bathtub will not change over time. For the convenience of calculation, we design an overflow pore. It ensures that the excess water outflow from the overflow pore, rather than outflow from the bathtub sides 
irregularly. Then we use numerical solution method to solve the steady temperature field.Generally speaking, water is a poor conductor of heat, and the heat transfer between liquid is mainly natural convection heat transfer. These points have been pointed out in our model . We extend the numerical solution of Seidel Gauss's method ${ }^{[4]}$ after absorbing the idea of Euler about fluid ${ }^{[3]}$. Euler method focuses on fixed point in space. So we divided the bath into $2 \mathrm{~cm} \times 2 \mathrm{~cm} \times 2 \mathrm{~cm}$ nodes. Then we can solve the fixed area nodes in the space.We regard the changes in the internal energy that caused by flowing water of the node as energy flows. So, we can get the temperature equation of each node in the discrete region by thermal equilibrium. We assume that the person is static. So its flowing form is laminar flow ${ }^{[3]}$ in the case of low water flow. The shape of spout from faucet in this distance below the surface is kept constant. Considering that the water is laminar, the temperature distribution of each layer is consistent in this distance. The temperature gradient between the layer and the layer is 0 . We perform a discrete calculation on the region.

We first set out the heat balance equation of internal nodes

$$
\begin{gathered}
t_{m, n}=\frac{\lambda\left(t_{m-1, n}+t_{m+1, n}+t_{m, n-1}+t_{m, n+1}\right)+h t_{a} \Delta x \Delta y}{4 \lambda+h t_{m, n} \Delta x \Delta y} \\
t_{m, n}=\frac{\frac{\lambda}{2}\left(t_{m-1, n}+t_{m+1, n}\right)+\lambda t_{m, n-1}+\frac{h \Delta x \Delta y}{2} t_{a}}{\lambda+\frac{\Delta x \Delta y}{2} h} \\
t_{m, n}=\frac{\frac{\lambda}{2}\left(t_{m-1, n}+t_{m, n-1}\right)+\frac{h \Delta x \Delta y}{4} t_{a}}{\lambda+\frac{\Delta x \Delta y}{4} h}
\end{gathered}
$$

$\lambda$---thermal conductivity of water, we can get it by searching information.

$h$---convective heat transfer coefficient, $h=\frac{N_{u} \lambda}{l_{1}}$.

$N_{u}$---Nusselt number, $N_{\mathrm{u}}=0.15\left(\mathrm{P}_{\mathrm{r}} G_{\mathrm{r}}\right)^{0.25}$.

$G_{r}$---Grashof number, $G r=\frac{g \alpha_{v} \Delta t l 1^{3}}{v^{2}}$.

After solving, we can get $h \approx 109 W /\left(m^{2} \bullet k\right)$.

In order to calculate the temperature distribution, we assume some original data (the temperature of bathtub is $35^{\circ} \mathrm{C}$, the temperature of hot water from faucet is $60^{\circ} \mathrm{C}$, the temperature on the surface of the human is $37^{\circ} \mathrm{C}$, the parameter of bathtub is $170 \mathrm{~cm} \times 80 \mathrm{~cm} \times 70 \mathrm{~cm}$ ) to perform an iterative operation. Then, we use Origin to plot the data we get.

\section{The result}

In order to obtain the mass flux of added hot water, we further simplify the model of bathtub. The Model has been given the temperature distribution of bathtub. We regard the bathtub as a lumped parameter model ${ }^{[1]}$ based on our model. We can get the average temperature of the bathtub $t_{p}$ by temperature distribution of model. We can calculate the mass flux of hot water after assuming the temperature of the water in the bathtub and the temperature of the added hot water.Then we can change the water temperature in bathtub by adjusting the hot water temperature and the mass flow of added hot water. This point is very consistent with the equation $t=f\left(x, y, z, W, t_{1} \ldots\right)$ given in model. We just simplify it in this model. The algorithm is shown below

Theory Evidence: $\rho c v \frac{d t}{d \tau}=\phi_{\text {in }}-\phi_{\text {out }}+v \bullet \dot{\phi}$

Since $\rho c v \frac{d t}{d \tau}=0$ and $\dot{\phi}=0$ we know $\phi_{\text {in }}=\phi_{\text {out }}$. 
$q_{m}$---mass flux.

$K$---convective coefficient of heat transfer.

$A^{\prime}$---radiating area of water surface, we can be obtained it by the size of the bathtub.

We assume that the average temperature of the bathtub $t_{p}=37^{\circ} \mathrm{C}$, the constant trickle of hot water from the faucet $t_{1}=55^{\circ} \mathrm{C}$, air temperature $t_{a}=25^{\circ} \mathrm{C}$.Substitute the values into equation (4.3 1), we can get the best mass flux of water

$$
q_{m}=0.061 \mathrm{Kg} / \mathrm{s}
$$

\section{Summary}

We through the establishment of model and the numerical solution method, we calculate the speed of hot water was added which can let people fell comfortable and can not waste too much energy, the result is

$$
q_{m}=0.061 \mathrm{Kg} / \mathrm{s}
$$

We fully describe the changes of temperature field in time and space by using equations. We creatively put forward the algorithm of equivalent internal heat source. It is convenient to calculate for us.But it is too complex to solve. So we can not give specific results and We assume that man is stationary, but it is not to be in reality.

\section{References}

[1]. [9787512371255]Yanfeng Liu, Zhengyang Gao, Xiujun Liang. HeatTransfer [M].Beijing: China Electric Power Press, 2015, 20-189.

[2]. [0559-9350(2004)02-0034-05]Zhenguo Zhao. Enthalpy DifferenceFormula for Calculating Heat Dissipation on Water Surface and its Use [J]. Journal of Hydraulic Engineering, 2004, (2):34-37.

[3]. [978-7-5083-5260-2]Songling Wang. Fluid Mechanics [M]. Beijing: China Electric Power Press, 2007, 31-32

[4]. [1000-2073(2010)01-0018-04]Yuqing Hu. A note on theGauss-Seidel-likemethod for Saddle Point Problems [J]. Suzhou University Journal of Medical Science, 2010, 01:19-21. 